\title{
Super-Compressibility Phenomenon
}

\author{
Kholmurad Khasanov \\ Gas and Wave Dynamics Department, Lomonosov Moscow State University, \\ Moscow, Russia \\ Email: kholkh@bk.ru
}

Received November 24, 2012; revised December 26, 2012; accepted January 6, 2013

\begin{abstract}
Super-compressibility and density jump of high-energy field in gases and in condensed matters are established as source of high density energy field in the nozzle with central cone. The nozzle is resonator and dynamic emitter of the fields in spiraled subsonic and supersonic jets. The values of super-accelerations are estimated as result of internal energy changing and decreasing during adiabatic expansion of the gas. Super accelerations in this field provide high energy radiation in continuous media. These phenomena can serve as basis for creation of new sources of energy.
\end{abstract}

Keywords: Supercompressibility; Superdensity Jump; Super High Density Field; Wave Spiral; Nozzle with a Central Cone

\section{Introduction}

Super high density energy field is detected in gas flowing from the nozzle with a central cone [1,2]. This nozzle is the resonator and dynamic emitter as in [3]. The emission of electromagnetic radiation of high energy during supercompressibility and superdensity jump in supersonic spiraled jet [4] is observed. The phenomenon of supercompressibility and superdensity jump in under-expanded submerged jet on output of the dynamic emitter results to the arising of super high density energy field in continuous media. In our experiments vapor jets form channels of stationary boundary layers with progressing strong compression of jet transverse and longitudinal cross-sections. Here we discuss the result of adiabatic expansion of the gas in dynamic emitter that results to the jet internal energy decreasing and its kinetic energy increasing. The measurements show that supercompressibility of supersonic jet has nonlinear character.

Super-compressibility, as result of super accelerations of the molecules of the jet, is accompanied by high energy radiation. It is close to multibubble compressibility at sonoluminescence phenomenon in liquid [5]. Sonoluminescence is the emission of short bursts of light from imploding bubbles in a liquid when excited by sound. But the phenomena discussed in our paper have significant differences from sonoluminescence and were found in the gas flows. Presented phenomena have nonlinear character. The evaluation of linear instability of the waves as sources of sound in a supersonic turbulent jet was discussed in [6]. They show that the noise generation process is not well modeled quantitatively by the linear theory. For the jet considered in their study, nonlinear effects are not only present, but they dominate the noise, as in our investigations. Their theoretical approach may be useful to explain our experiments.

Many open questions remain in the study of structured jets. Similar phenomena are observed during the jets emanating from young stellar objects. Of particular interest for astrophysics are issues related to the internal energy and internal structure of the jet [7]. Recent observations using Hubble Space Telescope and other highresolution platforms indicate that jets may content significant sub-radial structure $\left(\delta x<r_{\text {jet }}\right)$ with high Mach numbers $(M \sim 20)$, which implies that jets may be inherently heterogeneous. Thus experimental studies to date have shown that stable supersonic jets can propagate over long distances without energetic losses and without disruption of the structure. This material is accelerated in the gravitational field of the star, reaching supersonic velocities and producing a shockwave which emits in ultra-violet and X-rays regions [8].

Astrophysical jets are ubiquitous throughout the universe. They can be observed to emerge from proto-stellar objects, stellar X-ray binaries and super-massive black holes located at the center of active galaxies, and they are believed to originate from a central object that is surrounded by a magnetized accretion disc [8].

Usually they show that these jets propagating, maintaining their collimation over long distances is measured in units of the jet initial radius. The studied jets, as in our investigations, are quasi-isentropic, they are both lighter and heavier than the ambient and meet the two main scaling parameter requirements for proto-stellar jets: the 
ejection Mach number and the (ambient density)/(jet density) ratio [9].

In our investigations the ratio (ambient density)/(jet density) is equal at least 2 - 5 (see Section 3.1).

The lifetime of the molecules in pre-chamber of the dynamic emitter defines the super minimal intermolecular distance. The interaction leads to its high accelerations in the vicinity of the outlet of the annular nozzle. Heavy free ions, as $\left(\mathrm{OH}^{-}\right)$and $\left(\mathrm{N}_{2}^{+}\right)$, originating in the channels acquire relativistic accelerations. In $[10,11]$ was published: the force of attraction between molecules of oxygen $18.9 \times 10^{-11} \mathrm{~N}$; the acceleration of oxygen molecule $3.57 \times 10^{14} \mathrm{~m} / \mathrm{sec}^{2}$; the acceleration for hydrogen molecule $1.2 \times 10^{16} \mathrm{~m} / \mathrm{sec}^{2}$. Such super-accelerations are the cause of gas supercompressibility and collapse of its wave structure.

By the other way instead the jets with high Mach numbers $(M \sim 20)$ in present work we provide propagation without disruption of structured jets over long distances for low $(M \sim 1-3)$ Mach numbers and also for subsonic jets. Their propagation is accompanied with super high density energy field and cool effectively due to internal gas energy decrease and radiation losses.

\section{Theoretical Part}

\subsection{Supercompressibility of Continuous Media in the Adiabatic Approximation}

Super-compressibility and superdensity jump, as result of super-accelerations in spiraled supersonic jet, is accompanied with super high density energy field. The nozzle is the resonator and provides stationary wave structures in continuous media.

These structures form the stable boundary layers and emit super high density energy field, as summarized in $[10,11]$. The results of measurement of the characteristics of these fields are presented in this work.

The expansion process in the pre-chamber is not reversible and $(P, T)$ in different locations are different. But the behavior of gas within each sufficiently small volume is quite satisfactorily described by the adiabatic equation. One considers the expansion in the pre-chamber of the dynamic emitter as reversible adiabatic process. Enthalpy and internal energy of the gas are related by Equation (1).

Hereinafter the Equations (1)-(5) are given for a unit mass:

$$
h=e+p V .
$$

It is known [12] that internal energy $e$ as in (2) and enthalpy $h$ as in (3) are related with sound velocity $a$ as

$$
e=\frac{a^{2}}{\gamma(\gamma-1)},
$$

$$
h=\frac{a^{2}}{\gamma-1} .
$$

In (2)-(3) $\gamma$ is adiabatic index. Enthalpy we expressed from (1) and (3).

Author proposes to present it via sound velocity and adiabatic index as (4)

$$
h=e+p V=\frac{a^{2}}{(\gamma-1)}=\frac{a^{2}}{\gamma(\gamma-1)}+\frac{a^{2}}{\gamma} \text {. }
$$

Here in (4) we expressed

$$
\frac{a^{2}}{\gamma}=p V
$$

We investigate separately each summand in (1) and show that kinetic energy increasing in the conic cross section of pre-chamber occurs due to internal energy decreasing.

Phenomena of supercompressibility and superdensity jump arise as result of internal energy decreasing during adiabatic expansion of the gas. It leads to increasing of the force of intermolecular interacting and to a high acceleration of the molecules. The super-compressibility arises as a result of intensive interacting in the gas and its structures. Such molecular interaction generates the supercompressibility as in the vicinity of outlet of the nozzle and in the jets propagating over long distances without energetic losses and without disruption of the structure.

The force of intermolecular interacting bringing accelerated molecules on small distances. The molecules accelerated up to $10^{14}-10^{16} \mathrm{~m} / \mathrm{sec}^{2}[10,11]$ that provides electromagnetic field of super high density energy.

\subsection{Boundary Conditions in the Resonator}

In the first experiments we provided the field of super high density energy in vicinity of subsonic and supersonic under-expanded submerged flows, on output of dynamic emitter [3] as nozzle with a conic central body. The detailed design of the nozzle is shown in Figure 1. The length of the generated wave structure was equal to 10 diameters of outlet of the nozzle approximately. The phenomenon of supercompressibility and superdensity jump in under-expanded submerged jet on output of the dynamic emitter results to the arising of super high density energy field in continuous media. The nozzle was made from bronze and other metals. In case of water vapor jets the nozzle was made from composite material or meta-material as textolite.

Design of the nozzle in Figure 1 allows a longitudinal movement of the conic central body 1 inside the truncated cone 2 for a different disposition of its top relative to a cut of the nozzle. We have provided gas-dynamical 


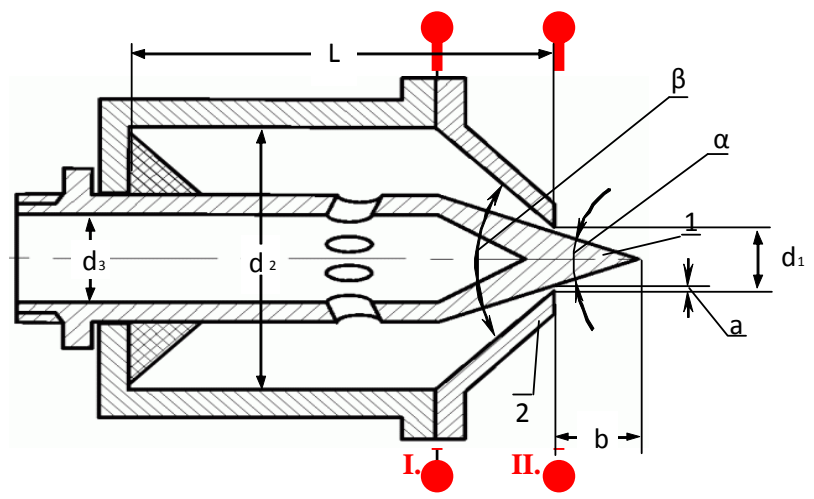

Figure 1. Design of the dynamic emitter with central cone. Here: 1 is central cone with diameter $d_{3}=25 \mathrm{~mm}$ and angle $\alpha=30^{\circ} ; 2$ is the truncated cone of the pre-chamber with diameter $d_{2}=60 \mathrm{~mm}$ and the angle $\beta=60^{\circ} ; d_{1}$ is a diameter of outlet of the nozzle; the annular gap $a=0.3-0.6 \mathrm{~mm}$; the external part o the internal cone $b=3-6 \mathrm{~mm}$; a length of pre-chamber $L=150 \mathrm{~mm}$. The dimensions are given in [1].

and energetic researches of other configurations of under-expanded submerged jets during their flowing from this nozzle at different operating conditions and dimensional parameters.

The optimal conditions for emergence of regular interacting subsonic and supersonic jets are given in [1]. We altered gas pressure and geometry of pre-chamber. Compressor provided filtered gas pressure in operating range $1.5-31 \mathrm{~atm}$. When the gas is flowing out into the atmosphere it acquires a configuration of strong pronounced compressed flow of spiral-twisted radiating wave structures. The structures consist of certain dimensions cells [4].

In the motionless volume from Figure 1, limited with sections I-I and II-II, in the absence of heat exchange with the environment, the total enthalpy is constant as (10)

$$
\left(h_{g e n}=\frac{W^{2}}{2}+h\right)=\text { const. }
$$

In (10) $W_{1}$ and $W_{2}$ are flow velocities in sections I-I and II-II comparatively and $h_{\text {gen }}$ is general enthalpy.

For sections I-I and II-II, we have the relation (11)

$$
\left(\frac{W_{1}^{2}}{2}+\frac{a_{36,1}^{2}}{\gamma_{1}\left(\gamma_{1}-1\right)}+\frac{a_{36,1}^{2}}{\gamma_{1}}\right)=\left(\frac{W_{2}^{2}}{2}+\frac{a_{36,2}^{2}}{\gamma_{2}\left(\gamma_{2}-1\right)}+\frac{a_{36,2}^{2}}{\gamma_{2}}\right) \text {. }
$$

Here also Equations (10) and (11) are given for a unit mass.

We have shown that in section II-II sum of decreasing of the pressure forces $(14531.0 \mathrm{~J})$ and of the internal en$\operatorname{ergy}(36326.5 \mathrm{~J})$ is $\Delta=36326.5+14531.0=50857.5 \mathrm{~J}$ and it is spent on the increasing of kinetic energy equal to $49,987 \mathrm{~J}$ and on the work against the friction forces. So, $(36326.5 / 49,987) \times 100=73 \%$ of the increasing in the kinetic energy in section II-II is due to the decreasing of internal energy of the gas.

The increasing of the kinetic energy is significant and is accompanied with a decreasing of the internal energy of gas and decreasing of the temperature. Sound velocity is reached at the critical section.

Previously G. N. Abramovich, S. A. Khristianovich, L. D. Landau, G. Black, V. G. Dulov, W. G. Pirumov, G. S. Roslyakov etc. considered the heat content (enthalpy) as a quantity which does divide on internal energy and $P V$. The calculations provide that contribution of the internal energy into kinetic energy of the jet is more than three times greater than energy of $P V$.

So, phenomena of supercompressibility, superdensity jump and the field of super high density energy arise due to decreasing of the internal energy of the continuous media.

\subsection{Estimations of Super Acceleration}

To estimate the acceleration of the molecules we use the model of hard elastic spheres and adjustment coefficients $a$ and $b$ of Van der Waals forces. Potential for interaction is given as (12):

$$
\varepsilon(r)=\left\{\begin{array}{cc}
\infty, & r<2 \cdot r_{S} ; \\
-\frac{\beta}{r^{m}}, & r \geq 2 \cdot r_{S},
\end{array}\right.
$$

Here $r_{S}$ is the radius of the cross section of collisions of gas molecules. The best approximation is achieved at $m=6$. Parameter $\beta$ is expressed through Avogadro's number $N_{A}$, and Van der Waals coefficients $a$ and $b$ as (13):

$$
\beta=\frac{9 a b}{4 \pi \cdot N_{A}^{3}}
$$

All parameters for some gases are given in Table 1.

The critical volume is given as (14) where radius of the cross section is given from (15) respectively:

$$
\begin{gathered}
V_{\text {crit }}=16 \pi \cdot N_{A} \cdot r_{S}^{3} \\
2 r_{S}=\sqrt[3]{\frac{V_{\text {crit }}}{2 \pi \cdot N_{A}}}
\end{gathered}
$$

The force of interaction that defines the super acceleration is given as (16):

$$
F=-\frac{\mathrm{d} \varepsilon}{\mathrm{d} r}=-\frac{m \beta}{r^{(m+1)}} .
$$

The acceleration one calculates from (16) for convenient mass of molecule. Heavy free ions, as $\left(\mathrm{OH}^{-}\right)$and $\left(\mathrm{N}_{2}^{+}\right)$, originating in the channels of the jets acquire relativistic accelerations. 
Table 1. Critical data and constant Van der Waals parameters.

\begin{tabular}{|c|c|c|c|c|c|c|}
\hline Gas & $P_{c}$, bar & $V_{\text {crit }}, \mathrm{m}^{3} / \mathrm{Kmol}$ & $\mathrm{T}_{\mathrm{c}},{ }^{\circ} \mathrm{K}$ & $a, b a r \cdot \mathrm{m}^{6} / \mathrm{Kmol}^{2}$ & $b, \mathrm{~m}^{3} / \mathrm{Kmol}$ & $R / N_{A} \cdot k$ \\
\hline $\mathrm{H}_{2}$ & 13.2 & 0.065 & 33.2 & 0.194 & 0.022 & 0.813 \\
\hline $\mathrm{H}_{2} \mathrm{O}$ & 225 & 0.055 & 647.3 & 5.65 & 0.031 & 0.602 \\
\hline $\mathrm{O}_{2}$ & 51.4 & 0.075 & 154.3 & 1.40 & 0.032 & 0.768 \\
\hline $\mathrm{N}_{2}$ & 34.8 & 0.090 & 126.0 & 1.39 & 0.039 & 0.782 \\
\hline
\end{tabular}

In $[10,11]$ was published that force of attraction between molecules of oxygen is $18.9 \times 10^{-11} \mathrm{~N}$; the acceleration of oxygen molecule is $3.57 \times 10^{14} \mathrm{~m} / \mathrm{sec}^{2}$; the acceleration for hydrogen molecule is $1.2 \times 10^{16} \mathrm{~m} / \mathrm{sec}^{2}$. Such super-accelerations are the cause of gas supercompressibility and collapse of its wave structure.

The values of super-accelerations are estimated as result of internal energy decreasing during adiabatic expansion of the gas in the field during the super-compressibility of the continuous media.

\section{Experimental Part}

In our experiments air and vapor-air jets form the channels of stationary boundary layers with progressing strong compression of jet in transverse and longitudinal cross-sections. For example, if the diameter of the nozzle cut was equal to $3 \mathrm{~mm}$, then the supercompressibility of jets was observed along the distance of $30 \mathrm{~mm}$ approximately. This unexpected phenomenon of a formation of a spiral twisted configuration may have various practical applications and it can lead to new tasks of development of gas-dynamical theory for his theoretical understanding in future. The jet with such configuration presents a new type of vortical flows as presented in Figure 2.

\subsection{Density Distribution in the Jet}

Results of visualizing of stationary boundary layers during their compressing along the coordinates of the jet show that jet has other density than ambient density. Author with Zakharova T. V. and Berezencev M. U. has held the measurement of the density distribution of the supersonic spiraled jet along its longitudinal and transverse coordinates. Method of supervision classification in the decision of aerodynamic problems [13] was provided.

The horizontal outflow of the jet in gravitational field was investigated. Distribution of density of the gas in $\left[\mathrm{Kg} / \mathrm{m}^{3}\right]$ is presented in Figure 3. It is scanned from the flooded area (left) to the nozzle (right).

Distribution of density of the gas in $\left[\mathrm{Kg} / \mathrm{m}^{3}\right]$ is presented in Figure 4 for transverse cross-section of the jet. It is scanned from bottom to top of the cross-section of horizontal outflow of the jet in gravitational field.
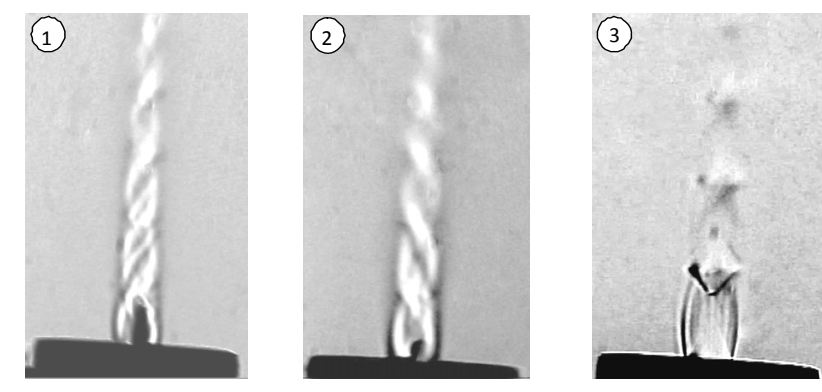

Figure 2. The shadowgraphs of formation the under-expanded submerged spiraled air jets. Here are right: (1) and left-twisted (2) rigid boundary layers as "horn of the unicorn". The direction of twist of air jet is changing versus different positions of the central cone outside the outlet of nozzle. A pressure in pre-chamber is 6 atm and temperature of surrounding air is $291^{\circ} \mathrm{K}$. On the right (3) the cone was on $4 \mathrm{~mm}$ inside the outlet of nozzle.

Here (in Figures 3 and 4) diameter of outlet of the nozzle was $3.6 \mathrm{~mm}$, the observed length of the jet was 25 $\mathrm{mm}$, air pressure in pre-chamber was $2.2-2.7 \mathrm{~atm}$, the critical cross-section on the nozzle output was $2 \mathrm{~mm}^{2}$. Jet density distributions along longitudinal and transverse coordinate of the jet are measured. The cross-section of the jet becomes blurred in gravity field during horizontal flowing of jet.

The ratio (ambient density)/(jet density) in our investigations is equal $2-5$.

\subsection{Flame Attraction to the Air Spiraled Jet}

Phenomenon of the candle flame attraction to the spiraled jet one observes. During gas flowing from the nozzle with a central cone the flame starts attracting and compressing with the jet (see Figure 5).

One can see from Figure 5 that in spiraled supersonic jet flowing from the nozzle with central cone the electromagnetic field with super high energy density is generated.

\subsection{Electromagnetic Characteristics of Spiral Vapor-Air Jet}

Taking in account our results with air flow we decided to use in the dynamic emitter water vapor for increasing the energy density [12]. During the flowing of the vapor-air 


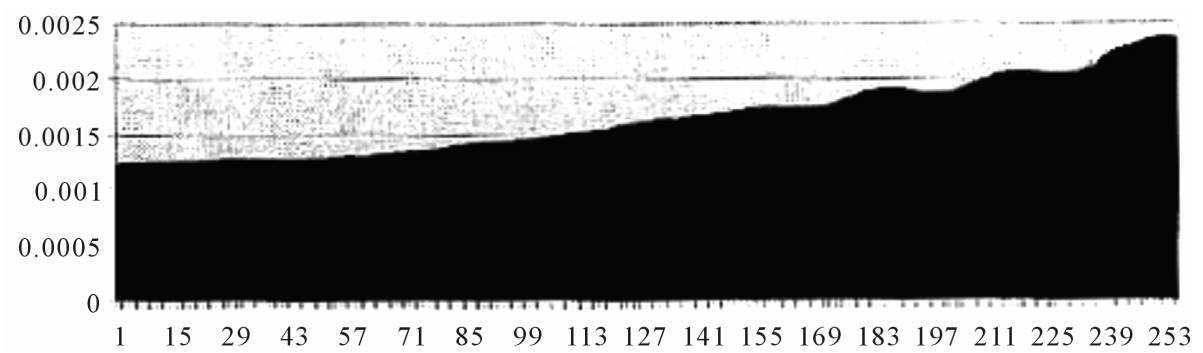

Figure 3. Density $p=p(L)$, in $\left[\mathrm{Kg} / \mathrm{m}^{3}\right]$, scanned from the flooded area (left) to the nozzle (right). Longitudinal coordinate $L$, in $\left[\mathrm{mm}^{*} \mathbf{0 . 1}\right]$, is on abscise axis of the draft.

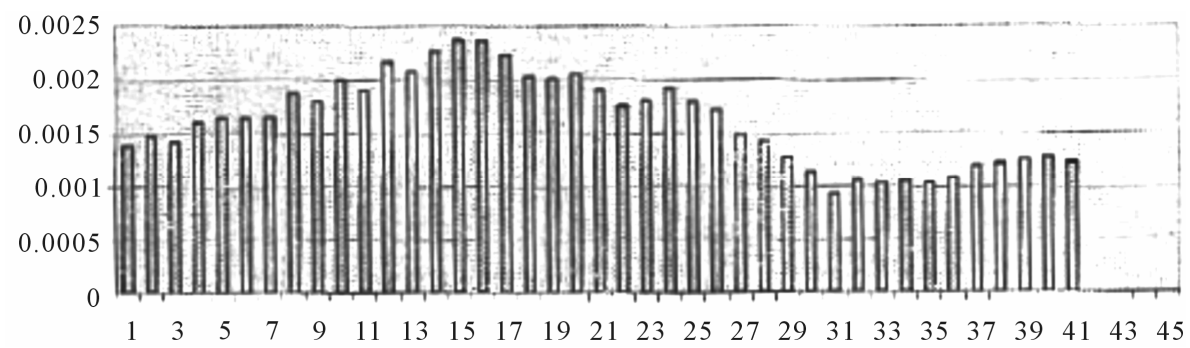

Figure 4. Density $p=p(D)$, in $\left[\mathrm{Kg} / \mathrm{m}^{3}\right]$, scanned from bottom (on left) up to top (on right) in vicinity the nozzle outlet. Transverse coordinate $D$, in $\left[\mathrm{mm}^{*} 0.1\right]$, is on abscise axis of the draft.

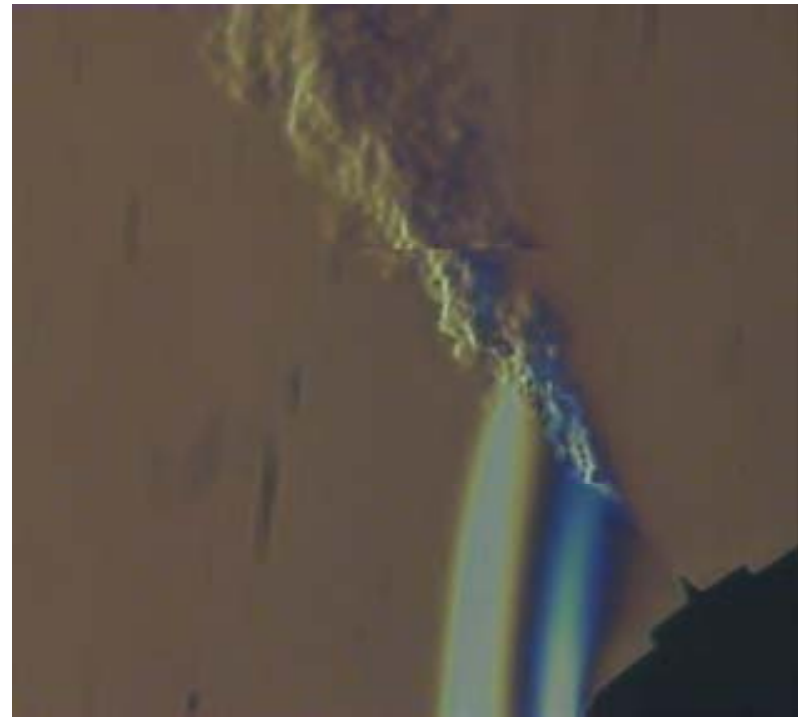

Figure 5. Candle flame attracting and compressing to the air spiral jet.

jet in the atmosphere the characteristics of plasma with more density of energy were measured. The emission of electromagnetic radiation from the X-ray to infrared spectrum has been found from initially neutral vapor [14]. The results below are obtained for jets as in Figure 6 .

High frequency voltage in jet in range $3-4 \mathrm{kV}$ for pre-chamber pressure $2-4 \mathrm{~atm}$ and $50-80 \mathrm{kV}$ at $10-12$ atm are measured at $393^{\circ} \mathrm{K}$ for both cases. Frequency of electromagnetic field in operating range $1.4 \mathrm{MHz}-1$ $\mathrm{GHz}$ for pre-chamber pressures in range $4-8 \mathrm{~atm}$ are detected.

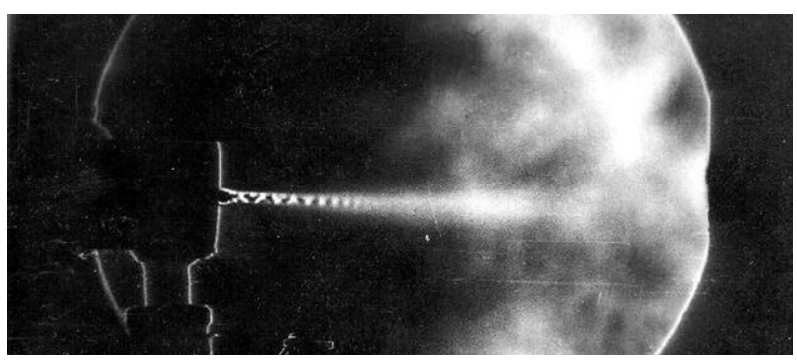

(a)

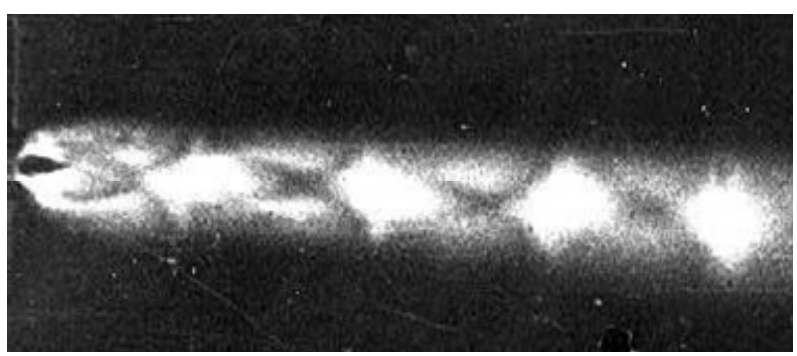

(b)

Figure 6. The shadowgraphs of the jets structure are presented for two cases of vapor-air jet flowing from the nozzle with a central cone. On the left pre-chamber pressure was provided $1.5 \mathrm{~atm}$, when is the beginning of the formation of regular structure in subsonic jet. On the right pre-chamber pressure was provided $31 \mathrm{~atm}$. Here is forming the structure of regular interacting shock waves in under-expanded supersonic jet.

Direct current voltage in the jet in range $80-100 \mathrm{kV}$ at pre-chamber pressure $10-12 \mathrm{~atm}, 393^{\circ} \mathrm{K}$ and electric field strength $10^{2} \mathrm{~V} / \mathrm{cm}$ around of the jet at distance $r=5$ $10 \mathrm{~m}$ are measured. Amplitude of electric current for HF 
field is in operating range $1 \mu \mathrm{A}-1 \mathrm{~mA}$ during short-circuit of the jet on the ground; at pressures 6-12 atm. Electrons density in different layers of the jet is estimated as $10^{9} \mathrm{~cm}^{-3}$ at distance $r=1 \mathrm{~m}$ in vapor-air jet. Electrons density near the central cone is $10^{15} \mathrm{~cm}^{-3}$ at distance $r=$ $10 \mathrm{~mm}-10 \mathrm{~cm}$ from the cone top. The accuracy of measurements was $0.5 \%$ at the multiple repetitions of the experiments.

The regular axial symmetric vortex structures in water vapor and air jets are obtained. These structures consist of the cells of certain dimensions. The type of structure and dimensions of its cells depends on the position of the central cone and pre-chamber pressure (see Figure 2).

The nozzle in Figure 6 provides boundary conditions for generation of this field. It is resonator and dynamic emitter of field with super high density energy in subsonic (Figure 6(a)) and supersonic (Figure 6(b)) jets.

\subsection{Air Jet Visualization with High Frequency Field}

To provide the study of the radiation characteristics during supercompressibility of supersonic jets, high frequency field was used as the mean for the jet visualization and its spectra registration. This type of discharge will occur when the strength or potential gradient of the electrical field around the conductor is high enough to form a conductive region, but not high enough to cause electrical breakdown or arcing to nearby objects. In our case the conductor is central cone in pre-chamber ( 1 in Figure 1).

The overview spectra of emission of radiation of the jets were obtained in region $\lambda=280-800 \mathrm{~nm}, \Delta \lambda=$ $0.293 \mathrm{~nm}$ with AVASPEC 2048. Detailed spectra of emission of radiation were obtained in optical region $\lambda=$ $383-527 \mathrm{~nm}, \Delta \lambda=0.083 \mathrm{~nm}$. The visualization was provided with electric discharge from HF generator, where frequency $f=268 \mathrm{kHz}$, output inductivity was $L=$ $21 \mathrm{mHn}$, generator power was $700-800 \mathrm{~W}$, current amplitude $I_{A}=0.5 \mathrm{~A}$, voltage amplitude $U_{A}=5 \mathrm{Kv}$.

High density energy field is detected in gas flowing from the nozzle with a central cone (Figure 7). The annual nozzle with central cone is dynamic emitter in which initially neutral gas is supplied. The super-compressibility and super-density jump arise as a result of high density field interacting within the continuous media.

In the third experiment with the same nozzle was supplied water vapor. The pressure of water vapor in prechamber was 2 - 12 atm, the temperature of vapor was $393^{\circ} \mathrm{K}$ in the pre-chamber and it decreased to $333^{\circ} \mathrm{K}$ on output of the dynamic emitter. The environment room temperature was $293^{\circ} \mathrm{K}$.

Beginning from 4 atm pressure, the high frequency field was detected from $1.4 \mathrm{MHz}$ up to $1 \mathrm{GHz}$ in spiral- twisted supersonic jets. The field arises in region both in and around of the jet.

In the figures one can see that the radiation in submerged jet has a regular character due to regularly interacting shock waves [15]. Therewith in Figure 8 one can see glowing space around the jet. In case of supplying water vapor supersonic jets start glowing in violet region and other in region of other short electromagnetic waves. Glowing in violet region is visually observed with the naked eye in dark conditions on approaching a conducting mass by $1-2 \mathrm{~m}$.

The increased brightness of discrete parts of the jet appears as a result of high electrons density. Water vapor provides plasma with more specific density of electrons energy that provides energy cumulation in small volumes

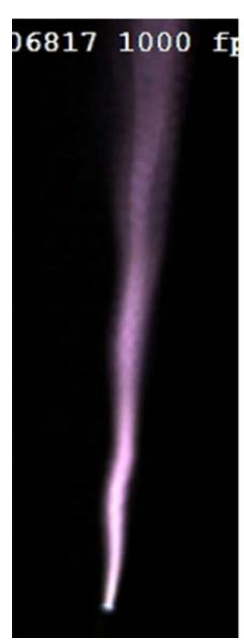

(a)

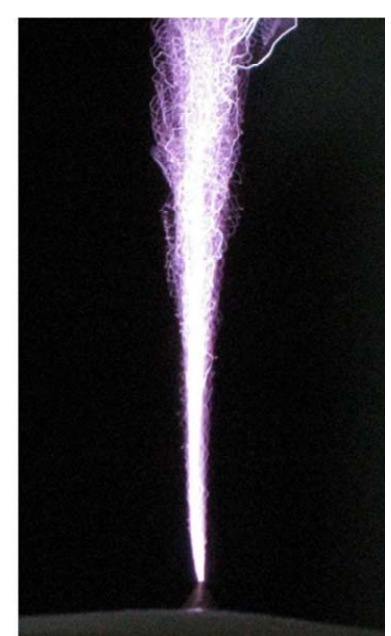

(b)

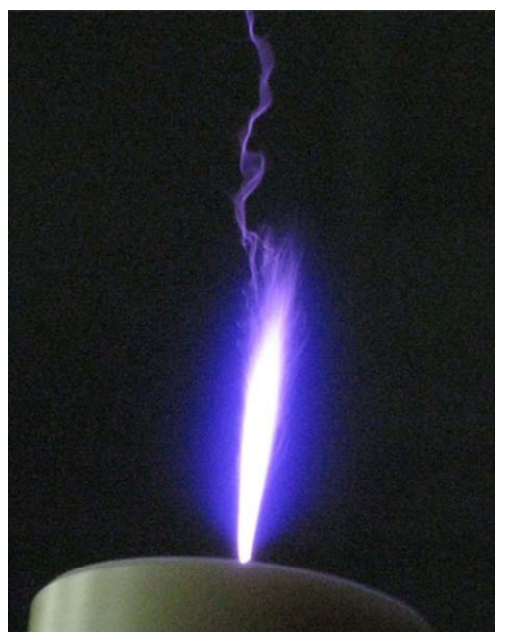

(c)

Figure 7. Small-scale wave structure formation. Here (a) is supersonic jet and (b) is subsonic jet. The pressure in the pre-chamber is for (a) $8 \mathrm{~atm}$ and less than $1 \mathrm{~atm}$ for (b). Exposure is $1 \mathrm{~ms}, 1000$ frames per second. Here (c) is halo formation in the vicinity of " plasma discharge channel. The pressure in pre-chamber for (c) is $\sim 4 \mathrm{~atm}$. 


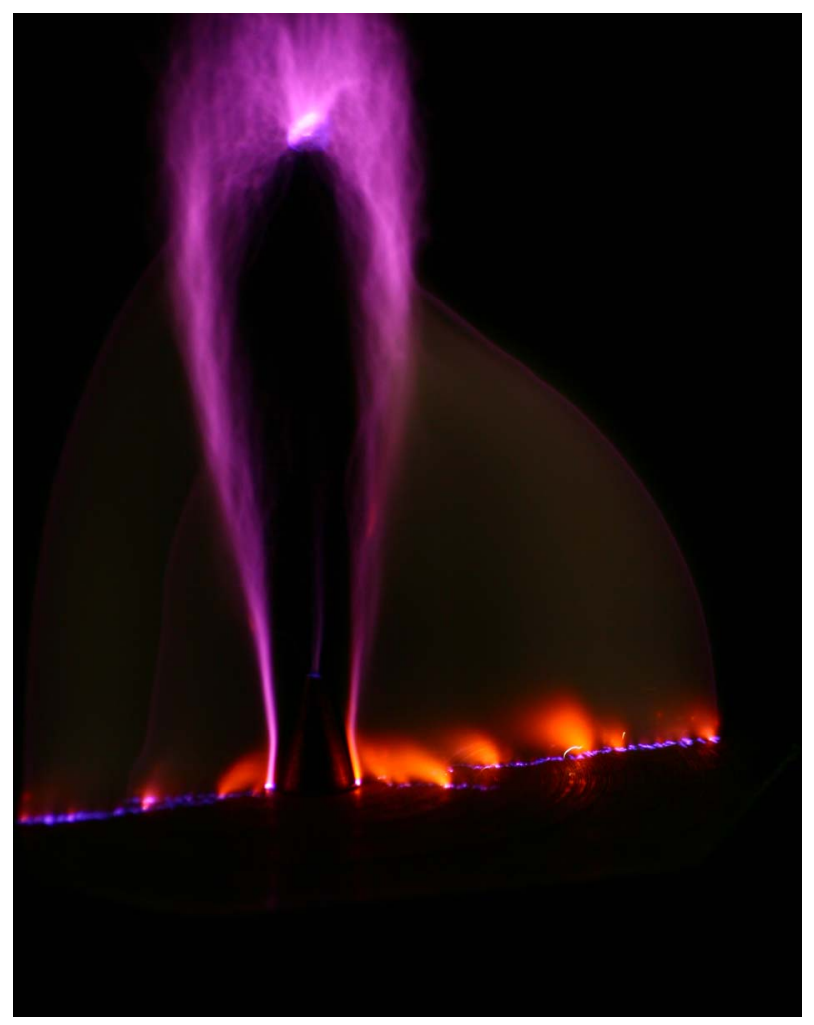

Figure 8. The antenna disposed on $10 \mathrm{~cm}$ over the top of cone. 1.4 - 1.6 MHz was supplied on the antenna. The metal plate and cone were grounded in the single-electrode scheme. Here is interaction of terahertz frequency fields of outer space with the jet. Against vector of gravitation.

and high energy radiation.

One can see that measured characteristics are enough for generation of radiation of high energy as result of super-compressibility of internal energy decreasing and kinetic energy increasing of supersonic spiral-twisted jets in the vapor-air structured flows.

\section{Conclusions}

- The phenomena of super-compressibility and density jump of high-energy field are established in gases and in condensed matters.

- These phenomena occur on small distances due to internal forces of an electrical nature are result of changing (decreasing) of the internal energy and super accelerations.

- The super accelerations provide high energy radiation in continuous media.

- The phenomena we establish can serve as a basis for the creation of new sources of high-density energy.

\section{Acknowledgements}

Author deeply appreciates to B. V. Melkoumian from A. M. Prokhorov General Physics Institute of RAS for comments, discussions and help in paper preparation.

Author gratefully acknowledges for the long-term support and consulting to M. V. Lomonosov Moscow State University rector, academician V. A. Sadovnichiy.

\section{REFERENCES}

[1] Kh. Khasanov, "Double Spiraled Supersonic Jet," Fluid Dynamics, Vol. 46, No. 3, 2011, pp. 433-436.

[2] Kh. Khasanov, "Bi-Spiral Switched Supersonic Jet Flow Escaping from a Circular Nozzle: Interaction with Metal and Polymer Screens, Infrared Radiation Phenomenon," International Conference: Fluxes and Structures in Fluids. Physics of Geospheres, Moscow, 24-27 June 2009, pp. 206-210.

[3] Kh. Khasanov and S. V. Petukhov, "Dynamic Emitter," PF Patent No. 2058196, 1996.

[4] Kh. Khasanov, "Visualization of Super-Compressibility in Supersonic Spiral-Twisted Jets," Physics Letters A, Vol. 376, No. 5, 2012, pp. 748-753.

[5] W. B. McNamara, Y. T. Didenko and K. S. Suslick, "Sonoluminescence Temperatures during Multi-Bubble Cavitation," Nature, Vol. 376, No. 5, 1999, pp. 772-775. doi:10.1016/j.physleta.2011.12.018

[6] K. Mohseni, T. Colonius and J. B. Freund, "An Evaluation of Linear Instability Waves as Sources of Sound in a Supersonic Turbulent Jet," Physical Fluids, Vol. 14, No. 10, 2002, pp. 3593-3600. doi:10.1063/1.1501545

[7] P. Hartigan, A. Frank, J. M. Foster, B. H. Wilde, M. Douglas, P. A. Rosen, R. F. Coker, B. E. Blue and J. F. Hansen, "Fluid Dynamics of Stellar Jets in Real Time: Third Epoch Hubble Space Telescope Images of HH 1, HH 34, and HH 47," Astrophysical Journal, Vol. 736, No. 1, 2011, p. 29. doi:10.1088/0004-637X/736/1/29

[8] D. W. Savin, et al., "The Impact of Recent Advances in Laboratory Astrophysics on Our Understanding of the Cosmos," Reports on Progress in Physics, Vol. 75, No. 3, 2012, pp. 1-36. doi:10.1088/0034-4885/75/3/036901

[9] D. Tordella, M. Belan, S. Massaglia, S. De Ponte, A. Mignone, E. Bodenschatz and A. Ferrari, "Astrophysical Jets: Insights into Long-Term Hydrodynamics," New Journal of Physics, Vol. 13, No. 4, 2011, 13 p. doi:10.1088/1367-2630/13/4/043011

[10] Kh. Khasanov, "Radiation of Bi-Spiral Switched Supersonic Jet Flow Escaping from an Annular Nozzle. Fluxes and Structures in Fluids. Physics of Geospheres-2009 (Abstracts)," Moscow, 24-27 June 2009.

[11] Kh. Khasanov, "Phenomena Taking Place at the Atomic Level under Certain Perturbation," Abstracts of 56th International Symposium on Molecular Spectroscopy, Ohio State University, Columbus, 2001, p. 125

[12] G. G. Chernyi, “Gas Dynamics,” Nauka, Moscow, 1988.

[13] T. V. Zakharova and M. U. Berezencev, "About a Method of Supervision Classification in the Decision of Aerodynamic Problems," Transactions of the XXIV International Seminar on Stability Problems for Stochastic Models, Riga: TTI, 2004, pp. 353-356. 
[14] Kh. Khasanov, "Emission of High Energy during SuperCompressibility of Supersonic Jets," Open Journal of Fluid Dynamics (OJFD), Vol. 2, 2012, pp. 172-179.

[15] Kh. Khasanov, "Visualization of Bi-Spiral Twisted Su- personic Gas Flow," The 8th Pacific Symposium of Flow Visualization and Image Processing, Moscow, 2011, p. 51. 